\title{
Synthesis of 3-(3-Methyl-1-aryl-1H-pyrazol-5-yl)-2H-2-chromen-2-one Derivatives via a One-Pot Three-Component Reaction
}

\author{
Abdolali Alizadeh,* Rashid Ghanbaripour \\ Department of Chemistry, Tarbiat Modares University, P.O. Box 14115-175, Tehran, Iran. E-mail: \\ aalizadeh@modares.ac.ir
}

SUPPORTING INFORMATION

\section{3-(3-Methyl-1-phenyl-1H-pyrazol-5-yl)-2H-chromen-2-one (3a).}

Yield: $181 \mathrm{mg}(60 \%)$; white powder; m.p. $=126-127^{\circ} \mathrm{C} . \mathrm{IR}(\mathrm{KBr}): 1724\left(\mathrm{CO}_{2}\right), 1602,1497$, and $1464(\mathrm{Ar}), 1106(\mathrm{C}-\mathrm{O}) \mathrm{cm}^{-1} .{ }^{1} \mathrm{H}$ NMR $\left(400.13 \mathrm{MHz}, \mathrm{CDCl}_{3}\right): \delta_{\mathrm{H}}=2.41\left(3 \mathrm{H}, \mathrm{s}, \mathrm{CH}_{3}\right), 6.63(1 \mathrm{H}, \mathrm{s}$, $\mathrm{CH}$ of pyrazole), $7.28\left(1 \mathrm{H}, \mathrm{d},{ }^{3} \mathrm{~J}_{\mathrm{HH}}=6.0 \mathrm{~Hz}, \mathrm{CH}_{\text {para }}\right.$ of $\left.\mathrm{Ph}\right), 7.33-7.35(4 \mathrm{H}, \mathrm{m}, \mathrm{CH}$ of $\mathrm{Ar}), 7.40-7.42$ (4H, m, CH of Ar), $7.48\left(1 \mathrm{H}, \mathrm{s}, \mathrm{CH}^{4}\right.$ of chromene), $7.55\left(1 \mathrm{H}, \mathrm{td},{ }^{3} J_{\mathrm{HH}}=8.0 \mathrm{~Hz},{ }^{4} J_{\mathrm{HH}}=1.6 \mathrm{~Hz}, \mathrm{CH}\right.$ of Ar). ${ }^{13} \mathrm{C}$ NMR $\left(100.6 \mathrm{MHz}, \mathrm{CDCl}_{3}\right): \delta_{\mathrm{C}}=13.56\left(\mathrm{CH}_{3}\right), 110.22\left(\mathrm{CH}^{3}\right.$ of pyrazole $), 116.68\left(\mathrm{CH}^{8}\right.$ of chromene), $118.62\left(\mathrm{C}^{3}\right.$ of chromene $), 118.88$ ( $\mathrm{C}^{4 \mathrm{a}}$ of chromene), $124.68\left(\mathrm{CH}^{6}\right.$ of chromene), $124.73\left(2 \mathrm{CH}_{\text {ortho }}\right.$ of $\left.\mathrm{Ph}\right), 127.70\left(\mathrm{CH}^{5}\right.$ of chromene $), 128.15\left(\mathrm{CH}_{\text {para }}\right.$ of $\left.\mathrm{Ph}\right), 129.30\left(2 \mathrm{CH}_{\text {meta }}\right.$ of $\mathrm{Ph}), 132.19\left(\mathrm{CH}^{7}\right.$ of chromene), $136.31\left(\mathrm{C}^{5}\right.$ of pyrazole $), 140.22\left(\mathrm{C}_{i p s o}-\mathrm{N}\right.$ of $\left.\mathrm{Ph}\right), 142.19\left(\mathrm{CH}^{4}\right.$ of chromene), 149.59 ( $\mathrm{C}^{3}$ of pyrazole) $153.56\left(\mathrm{C}^{8 \mathrm{a}}\right.$ of chromene), $158.97\left(\mathrm{CO}_{2}\right)$. MS (EI, $\left.70 \mathrm{eV}\right): \mathrm{m} / \mathrm{z}$ $(\%)=302\left(M^{+}, 100\right), 285(19), 273(62), 232(21), 204$ (34), 151 (25), 139 (28), 115 (33), $102(28)$, 91 (34), 77 (77). Anal. calcd for $\mathrm{C}_{19} \mathrm{H}_{14} \mathrm{~N}_{2} \mathrm{O}_{2}$ (302.33): C, 75.48; H, 4.67; N, 9.27\%. Found: $\mathrm{C}$, 75.37; H, 4.75; N, 9.20\%.

6-Bromo-3-(3-methyl-1-phenyl-1H-pyrazol-5-yl)-2H-chromen-2-one (3b). 
Yield: $278 \mathrm{mg}(73 \%)$. white powder. m.p. $=160-162{ }^{\circ} \mathrm{C} . \mathrm{IR}(\mathrm{KBr}): 1726\left(\mathrm{CO}_{2}\right), 1596,1541$, and $1493(\mathrm{Ar}), 1235$ and $1110(\mathrm{C}-\mathrm{O}) \mathrm{cm}^{-1} .{ }^{1} \mathrm{H} \mathrm{NMR}\left(400.13 \mathrm{MHz}, \mathrm{CDCl}_{3}\right): \delta_{\mathrm{H}}=2.42\left(3 \mathrm{H}, \mathrm{s}, \mathrm{CH}_{3}\right)$, $6.66\left(1 \mathrm{H}, \mathrm{s}, \mathrm{CH}\right.$ of pyrazole), $7.23\left(1 \mathrm{H}, \mathrm{d},{ }^{3} J_{\mathrm{HH}}=8.8 \mathrm{~Hz}, \mathrm{CH}^{8}\right.$ of chromene $), 7.36\left(1 \mathrm{H}, \mathrm{s}, \mathrm{CH}^{4}\right.$ of chromene), 7.41-7.42 (5H, m, $5 \mathrm{CH}$ of $\mathrm{Ph}), 7.45\left(1 \mathrm{H}, \mathrm{d},{ }^{4} \mathrm{~J}_{\mathrm{HH}}=2.0 \mathrm{~Hz}, \mathrm{CH}^{5}\right.$ of chromene), 7.63 $\left(1 \mathrm{H}, \mathrm{dd},{ }^{3} J_{\mathrm{HH}}=8.8 \mathrm{~Hz},{ }^{4} J_{\mathrm{HH}}=2.0 \mathrm{~Hz}, \mathrm{CH}^{7}\right.$ of chromene $) .{ }^{13} \mathrm{C} \mathrm{NMR}\left(100.6 \mathrm{MHz}, \mathrm{CDCl}_{3}\right): \delta_{\mathrm{C}}=$ $13.54\left(\mathrm{CH}_{3}\right), 110.52\left(\mathrm{CH}^{3}\right.$ of pyrazole $), 117.27(\mathrm{C}-\mathrm{Br}), 118.39\left(\mathrm{CH}^{8}\right.$ of chromene $), 119.92\left(\mathrm{C}^{3}\right.$ of chromene), 120.13 ( $\mathrm{C}^{4 \mathrm{a}}$ of chromene), $124.79\left(2 \mathrm{CH}_{\text {ortho }}\right.$ of $\left.\mathrm{Ph}\right), 127.93\left(\mathrm{CH}_{\text {para }}\right.$ of $\left.\mathrm{Ph}\right), 129.42(2$ $\mathrm{CH}_{\text {meta }}$ of $\left.\mathrm{Ph}\right), 130.25\left(\mathrm{CH}^{5}\right.$ of chromene), $134.84\left(\mathrm{CH}^{7}\right.$ of chromene $), 135.78\left(\mathrm{C}^{5}\right.$ of pyrazole $)$, $140.07\left(\mathrm{C}_{i p s o}-\mathrm{N}\right.$ of $\left.\mathrm{Ph}\right), 140.43\left(\mathrm{CH}^{4}\right.$ of chromene), $149.71\left(\mathrm{C}^{3}\right.$ of pyrazole $), 152.30\left(\mathrm{C}^{8 \mathrm{a}}\right.$ of chromene), $158.29\left(\mathrm{CO}_{2}\right)$. MS (EI, $\left.70 \mathrm{eV}\right): \mathrm{m} / \mathrm{z}(\%)=382(M+2,33), 380\left(M^{+}, 34\right), 241(40), 188$ (100), 173 (43), 161 (20), 145 (40), 118 (27), 107 (26), 91 (24), 77 (40). Anal. calcd for $\mathrm{C}_{19} \mathrm{H}_{13} \mathrm{BrN}_{2} \mathrm{O}_{2}$ (381.23): C, 59.86; H, 3.44; N, 7.35\%. Found: C, 59.96; H, 3.37; N, 7.31\%.

\section{8-Methoxy-3-(3-methyl-1-phenyl-1H-pyrazol-5-yl)-2H-chromen-2-one (3c).}

Yield: $229 \mathrm{mg}$ (69\%). yellow oil. IR (KBr): $1720\left(\mathrm{CO}_{2}\right), 1601,1484$ and $1449(\mathrm{Ar}), 1276$ and 1100 $(\mathrm{C}-\mathrm{O}) \mathrm{cm}^{-1} .{ }^{1} \mathrm{H}$ NMR $\left(400.13 \mathrm{MHz}, \mathrm{CDCl}_{3}\right): \delta_{\mathrm{H}}=2.41\left(3 \mathrm{H}, \mathrm{s}, \mathrm{CH}_{3}\right), 3.97\left(3 \mathrm{H}, \mathrm{s}, \mathrm{OCH}_{3}\right), 6.63(1 \mathrm{H}$, s, CH of pyrazole), $6.90\left(1 \mathrm{H}, \mathrm{dd},{ }^{3} J_{\mathrm{HH}}=8.0 \mathrm{~Hz},{ }^{4} J_{\mathrm{HH}}=1.2 \mathrm{~Hz}, \mathrm{CH}^{7}\right.$ of chromene), $7.09(1 \mathrm{H}, \mathrm{dd}$, ${ }^{3} J_{\mathrm{HH}}=8.0 \mathrm{~Hz},{ }^{4} J_{\mathrm{HH}}=1.2 \mathrm{~Hz}, \mathrm{CH}^{5}$ of chromene), $7.19\left(1 \mathrm{H}, \mathrm{t},{ }^{3} J_{\mathrm{HH}}=8.0 \mathrm{~Hz}, \mathrm{CH}^{6}\right.$ of chromene), 7.32-7.41 (5H, m, $5 \mathrm{CH}$ of $\mathrm{Ph}), 7.45\left(1 \mathrm{H}, \mathrm{s}, \mathrm{CH}^{4}\right.$ of chromene). ${ }^{13} \mathrm{C} \mathrm{NMR}\left(100.6 \mathrm{MHz}, \mathrm{CDCl}_{3}\right): \delta_{\mathrm{C}}$ $=13.55\left(\mathrm{CH}_{3}\right), 56.27\left(\mathrm{OCH}_{3}\right), 110.26\left(\mathrm{CH}^{3}\right.$ of pyrazole $), 113.92\left(\mathrm{CH}^{7}\right.$ of chromene $), 119.12\left(\mathrm{C}^{3}\right.$ of chromene), 119.23 ( $\mathrm{C}^{4 \mathrm{a}}$ of chromene), $119.46\left(\mathrm{CH}^{5}\right.$ of chromene), $124.54\left(\mathrm{CH}^{6}\right.$ of chromene), $124.76\left(2 \mathrm{CH}_{\text {ortho }}\right.$ of $\left.\mathrm{Ph}\right), 127.66\left(\mathrm{CH}_{\text {para }}\right.$ of $\left.\mathrm{Ph}\right), 129.28\left(2 \mathrm{CH}_{\text {meta }}\right.$ of $\left.\mathrm{Ph}\right), 136.31$ ( $\mathrm{C}^{5}$ of pyrazole), $140.19\left(\mathrm{C}_{\text {ipso }}-\mathrm{N}\right.$ of $\left.\mathrm{Ph}\right), 142.35\left(\mathrm{CH}^{4}\right.$ of chromene), $143.23\left(\mathrm{C}^{8 \mathrm{a}}\right.$ of chromene $), 147.08\left(\mathrm{C}^{3}\right.$ of pyrazole), $149.56\left(\mathrm{C}-O C H_{3}\right), 158.41\left(\mathrm{CO}_{2}\right)$. MS (EI, $\left.70 \mathrm{eV}\right): \mathrm{m} / \mathrm{z}(\%)=332\left(M^{+}, 100\right), 315(30)$, 
303 (20), 261 (11), 191 (11), 91 (27), 84 (52), 76 (31), 51 (43). Anal. calcd for $\mathrm{C}_{20} \mathrm{H}_{16} \mathrm{~N}_{2} \mathrm{O}_{3}$ (332.36): C, 72.28; H, 4.85; N, 8.43\%. Found: C, 72.45; H, 4.97; N, 8.30\%.

\section{6,8-Dibromo-3-(3-methyl-1-phenyl-1H-pyrazol-5-yl)-2H-chromen-2-one (3d).}

Yield: $409 \mathrm{mg}$ (89\%). yellow powder. m.p. $=263-265^{\circ} \mathrm{C}$. IR (KBr): $3058(\mathrm{CH}$ of aromatic), 1738 $\left(\mathrm{CO}_{2}\right), 1595,1498$ and $1451(\mathrm{Ar}), 1396,1364(\mathrm{Me}), 1247$ and $1098(\mathrm{C}-\mathrm{O}) \mathrm{cm}^{-1} .{ }^{1} \mathrm{H}$ NMR (400.13 $\left.\mathrm{MHz}, \mathrm{CDCl}_{3}\right): \delta_{\mathrm{H}}=2.42\left(3 \mathrm{H}, \mathrm{s}, \mathrm{CH}_{3}\right), 6.69(1 \mathrm{H}, \mathrm{s}, \mathrm{CH}$ of pyrazole $), 7.29\left(1 \mathrm{H}, \mathrm{s}, \mathrm{CH}^{4}\right.$ of chromene $)$, $7.38\left(1 \mathrm{H}, \mathrm{d},{ }^{4} J_{\mathrm{HH}}=2.0 \mathrm{~Hz}, \mathrm{CH}^{5}\right.$ of chromene $), 7.40-7.43(5 \mathrm{H}, \mathrm{m}, 5 \mathrm{CH}$ of $\mathrm{Ph}), 7.88\left(1 \mathrm{H}, \mathrm{d},{ }^{4} J_{\mathrm{HH}}=\right.$ $2.0 \mathrm{~Hz}, \mathrm{CH}^{7}$ of chromene). ${ }^{13} \mathrm{C}$ NMR $\left(100.6 \mathrm{MHz}, \mathrm{CDCl}_{3}\right): \delta_{\mathrm{C}}=13.54\left(\mathrm{CH}_{3}\right), 110.83\left(\mathrm{CH}^{3}\right.$ of pyrazole), $111.19\left(\mathrm{C}^{8}-\mathrm{Br}\right), 117.17\left(\mathrm{C}^{3}\right.$ of chromene), 120.52 ( $\mathrm{C}^{4 \mathrm{a}}$ of chromene), $120.86\left(\mathrm{C}^{6}-\mathrm{Br}\right)$, $124.86\left(2 \mathrm{CH}_{\text {ortho }}\right.$ of $\left.\mathrm{Ph}\right), 128.09\left(\mathrm{CH}_{\text {para }}\right.$ of $\left.\mathrm{Ph}\right), 129.44\left(\mathrm{CH}^{5}\right.$ of chromene $), 129.51\left(2 \mathrm{CH}_{\text {meta }}\right.$ of $\mathrm{Ph}), 135.25$ ( $\mathrm{C}^{5}$ of pyrazole), $137.46\left(\mathrm{CH}^{4}\right.$ of chromene $), 139.87\left(\mathrm{CH}^{7}\right.$ of chromene $), 139.92\left(\mathrm{C}_{\text {ipso- }}\right.$ $\mathrm{N}$ of $\mathrm{Ph}), 149.26\left(\mathrm{C}^{3}\right.$ of pyrazole), $149.80\left(\mathrm{C}^{8 \mathrm{a}}\right.$ of chromene), $157.39\left(\mathrm{CO}_{2}\right) . \mathrm{MS}(\mathrm{EI}, 70 \mathrm{eV}): \mathrm{m} / \mathrm{z}$ $(\%)=462(M+4,52), 460(M+2,100), 458\left(M^{+}, 52\right), 431(38), 297(36), 201(30), 139(22), 77$ (79), 51 (31). Anal. calcd for $\mathrm{C}_{19} \mathrm{H}_{12} \mathrm{Br}_{2} \mathrm{~N}_{2} \mathrm{O}_{2}$ (460.12): C, 49.60; H, 2.63; N, 6.09\%. Found: $\mathrm{C}$, 49.74; H, 2.58; N, 6.18\%.

\section{6,8-Dibromo-3-[3-methyl-1-(4-methylphenyl)-1H-pyrazol-5-yl]-2H-chromen-2-one (3e).}

Yield: $398 \mathrm{mg}(84 \%)$. white powder. m.p. $=258-261{ }^{\circ} \mathrm{C}$. IR $(\mathrm{KBr}): 1739\left(\mathrm{CO}_{2}\right), 1553,1515$ and $1452(\mathrm{Ar}), 1284(\mathrm{C}-\mathrm{O}) \mathrm{cm}^{-1} .{ }^{1} \mathrm{H}$ NMR $\left(400.13 \mathrm{MHz}, \mathrm{CDCl}_{3}\right): \delta_{\mathrm{H}}=2.40\left(3 \mathrm{H}, \mathrm{s}, \mathrm{CH}_{3}\right), 2.41(3 \mathrm{H}, \mathrm{s}$, $\left.\mathrm{CH}_{3}\right), 6.66(1 \mathrm{H}, \mathrm{s}, \mathrm{CH}$ of pyrazole $), 7.21\left(2 \mathrm{H}, \mathrm{d},{ }^{3} \mathrm{~J}_{\mathrm{HH}}=8.4 \mathrm{~Hz}, \mathrm{CH}\right.$ of Ar$), 7.26\left(2 \mathrm{H}, \mathrm{d},{ }^{3} J_{\mathrm{HH}}=8.4\right.$ $\mathrm{Hz}, \mathrm{CH}$ of Ar), $7.30\left(1 \mathrm{H}, \mathrm{s}, \mathrm{CH}^{4}\right.$ of chromene), $7.39\left(1 \mathrm{H}, \mathrm{d},{ }^{4} \mathrm{~J}_{\mathrm{HH}}=2.0 \mathrm{~Hz}, \mathrm{CH}^{5}\right.$ of chromene), 7.88 $\left(1 \mathrm{H}, \mathrm{d},{ }^{4} J_{\mathrm{HH}}=2.0 \mathrm{~Hz}, \mathrm{CH}^{7}\right.$ of chromene). ${ }^{13} \mathrm{C} \mathrm{NMR}\left(100.6 \mathrm{MHz}, \mathrm{CDCl}_{3}\right): \delta_{\mathrm{C}}=13.53\left(\mathrm{CH}_{3}\right.$ of pyrazole $), 21.17\left(\mathrm{CH}_{3}\right.$ of chromene $), 110.55\left(\mathrm{CH}^{3}\right.$ of pyrazole $), 111.16\left(\mathrm{C}^{8}-\mathrm{Br}\right), 117.11\left(\mathrm{C}^{3}\right.$ of chromene), 120.66 ( $\mathrm{C}^{4 \mathrm{a}}$ of chromene), $120.92\left(\mathrm{C}^{6}-\mathrm{Br}\right), 124.71(2 \mathrm{CH}$ of $\mathrm{Ar}), 129.44\left(\mathrm{CH}^{5}\right.$ of 
chromene), 130.06 (2 $\mathrm{CH}$ of $\mathrm{Ar}), 135.19$ ( $\mathrm{C}^{5}$ of pyrazole), 137.38 ( $\mathrm{CH}^{4}$ of chromene), 137.49 (C-N of $\mathrm{Ar}), 138.02$ ( $\mathrm{C}-\mathrm{CH}_{3}$ of $\left.\mathrm{Ar}\right), 139.75\left(\mathrm{CH}^{7}\right.$ of chromene), $149.47\left(\mathrm{C}^{3}\right.$ of pyrazole), $149.54\left(\mathrm{C}^{8 \mathrm{a}}\right.$ of chromene), $156.43\left(\mathrm{CO}_{2}\right)$. MS (EI, $\left.70 \mathrm{eV}\right): \mathrm{m} / \mathrm{z}(\%)=476(M+4,51), 474(M+2,100), 472\left(M^{+}\right.$, 59), 445 (28), 216 (15), 189 (11), 139 (16), 91 (45), 65 (41). Anal. calcd for $\mathrm{C}_{20} \mathrm{H}_{14} \mathrm{Br}_{2} \mathrm{~N}_{2} \mathrm{O}_{2}$ (474.15): C, 50.66; H, 2.98; N, 5.91\%. Found: C, 50.61; H, 2.87; N, 6.02\%.

\section{6,8-Dichloro-3-(3-methyl-1-phenyl-1H-pyrazol-5-yl)-2H-chromen-2-one (3f).}

Yield: $297 \mathrm{mg}(80 \%)$. white powder. m.p. $=244-246{ }^{\circ} \mathrm{C}$. IR $(\mathrm{KBr}): 1737\left(\mathrm{CO}_{2}\right), 1565,1498$ and $1456(\mathrm{Ar}), 1243(\mathrm{C}-\mathrm{O}) \mathrm{cm}^{-1} .{ }^{1} \mathrm{H} \mathrm{NMR}\left(400.13 \mathrm{MHz}, \mathrm{CDCl}_{3}\right): \delta_{\mathrm{H}}=.2 .42\left(3 \mathrm{H}, \mathrm{s}, \mathrm{CH}_{3}\right), 6.69(1 \mathrm{H}, \mathrm{s}$, $\mathrm{CH}$ of pyrazole), $7.20\left(1 \mathrm{H}, \mathrm{d},{ }^{4} \mathrm{~J}_{\mathrm{HH}}=2.4 \mathrm{~Hz}, \mathrm{CH}^{5}\right.$ of chromene $), 7.32\left(1 \mathrm{H}, \mathrm{s}, \mathrm{CH}^{4}\right.$ of chromene), 7.32-7.43 $(5 \mathrm{H}, \mathrm{m}, 5 \mathrm{CH}$ of $\mathrm{Ph}), 7.58\left(1 \mathrm{H}, \mathrm{d},{ }^{4} J_{\mathrm{HH}}=2.4 \mathrm{~Hz}, \mathrm{CH}^{7}\right.$ of chromene $) .{ }^{13} \mathrm{C}$ NMR $(100.6$ $\left.\mathrm{MHz}, \mathrm{CDCl}_{3}\right): \delta_{\mathrm{C}}=13.54\left(\mathrm{CH}_{3}\right), 110.80\left(\mathrm{CH}^{3}\right.$ of pyrazole $), 120.41\left(\mathrm{C}^{8}-\mathrm{Cl}\right), 120.59\left(\mathrm{C}^{3}\right.$ of chromene), 122.56 ( $\mathrm{C}^{4 \mathrm{a}}$ of chromene), 124.86 (2 $\mathrm{CH}_{\text {ortho }}$ of $\left.\mathrm{Ph}\right), 125.75$ ( $\mathrm{CH}^{5}$ of chromene), 128.07 $\left(\mathrm{CH}_{\text {para }}\right.$ of $\left.\mathrm{Ph}\right), 129.49$ (2 $\mathrm{CH}_{\text {meta }}$ of $\left.\mathrm{Ph}\right), 128.81\left(\mathrm{C}^{6}-\mathrm{Cl}\right), 131.94\left(\mathrm{CH}^{7}\right.$ of chromene), $135.31\left(\mathrm{C}^{5}\right.$ of pyrazole), $139.94\left(\mathrm{C}_{i p s o}-\mathrm{N}\right.$ of $\left.\mathrm{Ph}\right), 139.99\left(\mathrm{CH}^{4}\right.$ of chromene $), 147.74$ ( $\mathrm{C}^{8 \mathrm{a}}$ of chromene), $149.79\left(\mathrm{C}^{3}\right.$ of pyrazole), $157.30\left(\mathrm{CO}_{2}\right)$. MS (EI, $\left.70 \mathrm{eV}\right): m / z(\%)=374(M+4,9), 372(M+2,52), 370\left(M^{+}, 81\right)$, 353 (20), 341 (32), 297 (49), 241 (56), 201 (31), 188 (29), 174 (65), 77 (100), 51 (47). Anal. calcd for $\mathrm{C}_{19} \mathrm{H}_{12} \mathrm{Cl}_{2} \mathrm{~N}_{2} \mathrm{O}_{2}$ (371.22): C, 61.48; H, 3.26; N, 7.55\%. Found: C, 61.44; H, 3.41; N, 7.69\%.

\section{3-[(Z)-1-Hydroxy-3-oxo-1-butenyl]-2H-chromen-2-one (4a).}

Yield: $207 \mathrm{mg}(90 \%)$. green-yellow powder. m.p. $=148-150{ }^{\circ} \mathrm{C} .{ }^{1} \mathrm{H}$ NMR $\left(500.13 \mathrm{MHz}, \mathrm{CDCl}_{3}\right)$ : $\delta_{\mathrm{H}}=2.26\left(\mathrm{~s}, 3 \mathrm{H}, \mathrm{CH}_{3}\right), 7.02\left(\mathrm{~s}, 1 \mathrm{H}, \mathrm{CH}\right.$ of vinylic), $7.33\left(\mathrm{~d},{ }^{3} \mathrm{~J}=7.6 \mathrm{~Hz}, 1 \mathrm{H}, \mathrm{CH}\right.$ of Ar), $7.36\left(\mathrm{~d},{ }^{3} \mathrm{~J}\right.$ $=7.6 \mathrm{~Hz}, 1 \mathrm{H}, \mathrm{CH}$ of Ar), $7.63\left(\mathrm{t},{ }^{3} \mathrm{~J}=7.6 \mathrm{~Hz}, 2 \mathrm{H}, 2 \mathrm{CH}\right.$ of Ar), 8.64 (s, 1H, Ch of chromene), 15.86 $(\mathrm{s}, 1 \mathrm{H}, \mathrm{OH}) \cdot{ }^{13} \mathrm{C} \mathrm{NMR}\left(125.7 \mathrm{MHz}, \mathrm{CDCl}_{3}\right): \delta_{\mathrm{C}}=27.30\left(\mathrm{CH}_{3}\right), 101(\mathrm{CH}$ of vinylic), $116.57(\mathrm{CH}$ of 
$\mathrm{Ar}), 118.53\left(\mathrm{C}^{3}\right), 120.63\left(\mathrm{C}^{4 \mathrm{a}}\right), 124.94(\mathrm{CH}$ of $\mathrm{Ar}), 129.55(\mathrm{CH}$ of $\mathrm{Ar}), 133.96(\mathrm{CH}$ of $\mathrm{Ar}), 145.43$

$\left(\mathrm{CH}^{4}\right), 154.40\left(\mathrm{C}^{8 \mathrm{a}}\right), 158.03\left(\mathrm{CO}_{2}\right), 171.97(\mathrm{C}-\mathrm{OH}), 199.69(\mathrm{CO})$. 


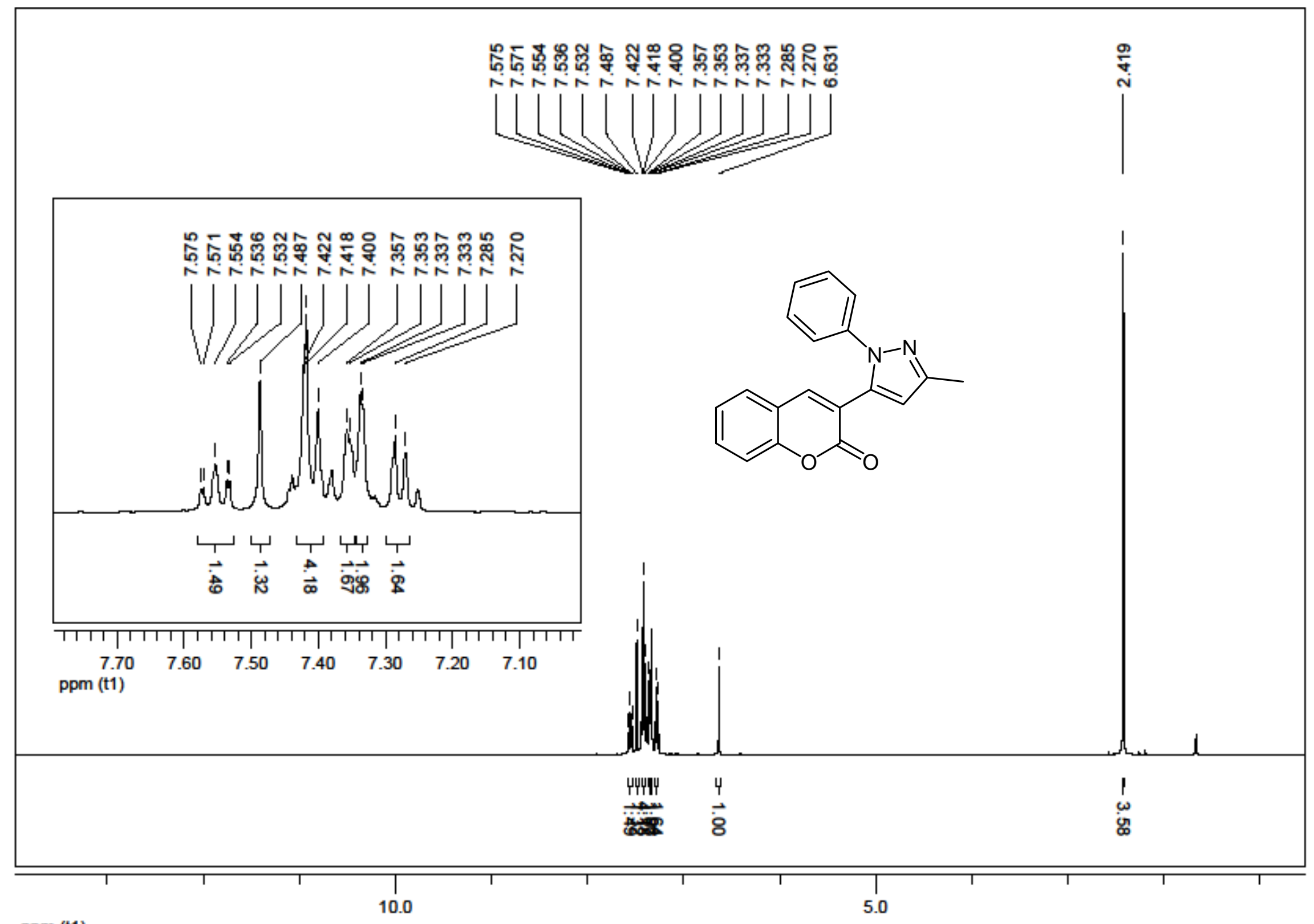

ppm (t1)

Figure $\mathrm{S} 1{ }^{1} \mathrm{H}$ NMR of $\mathbf{3 a}$ 


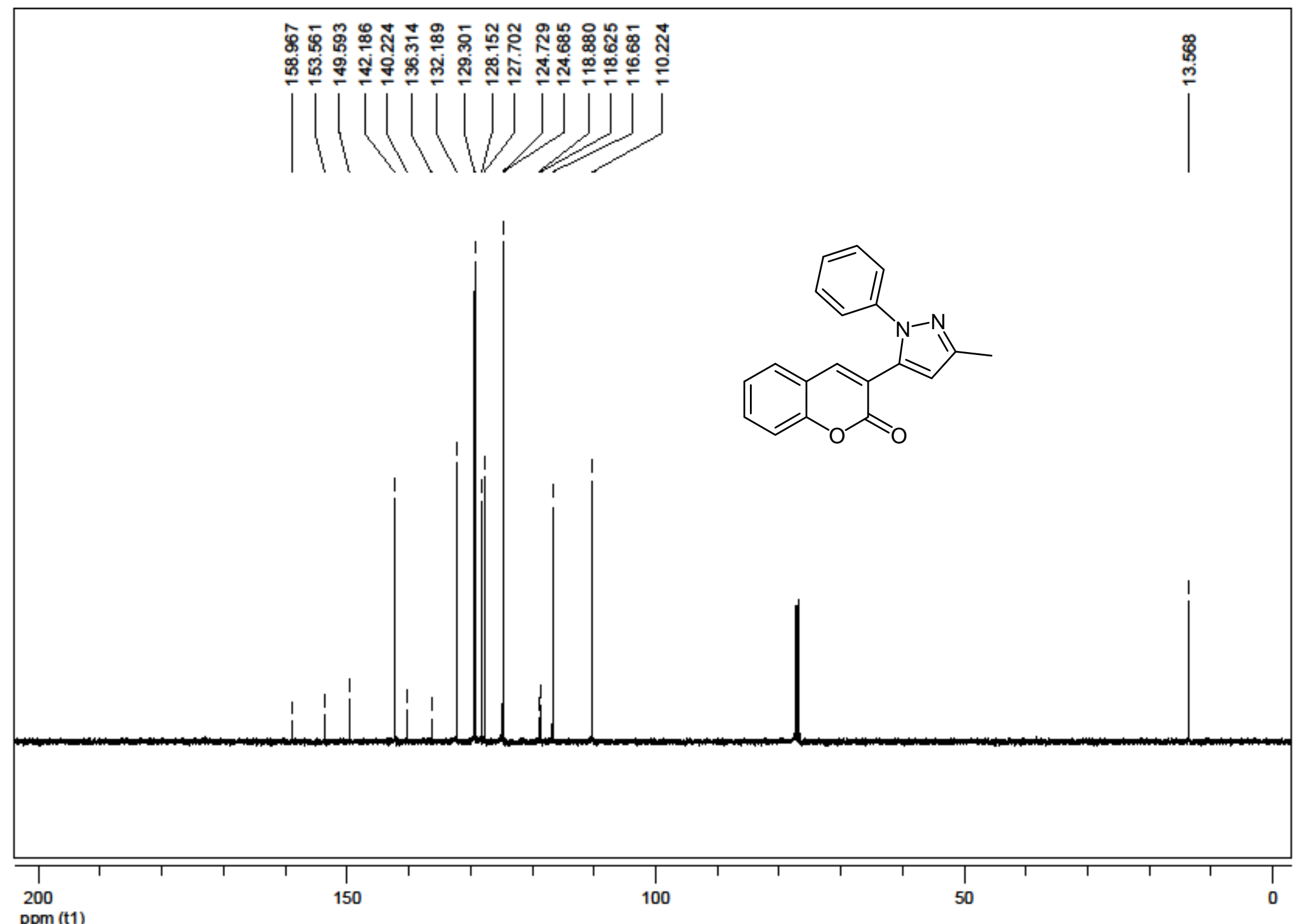

Figure $\mathrm{S} 2{ }^{13} \mathrm{C}$ NMR of $\mathbf{3 a}$ 


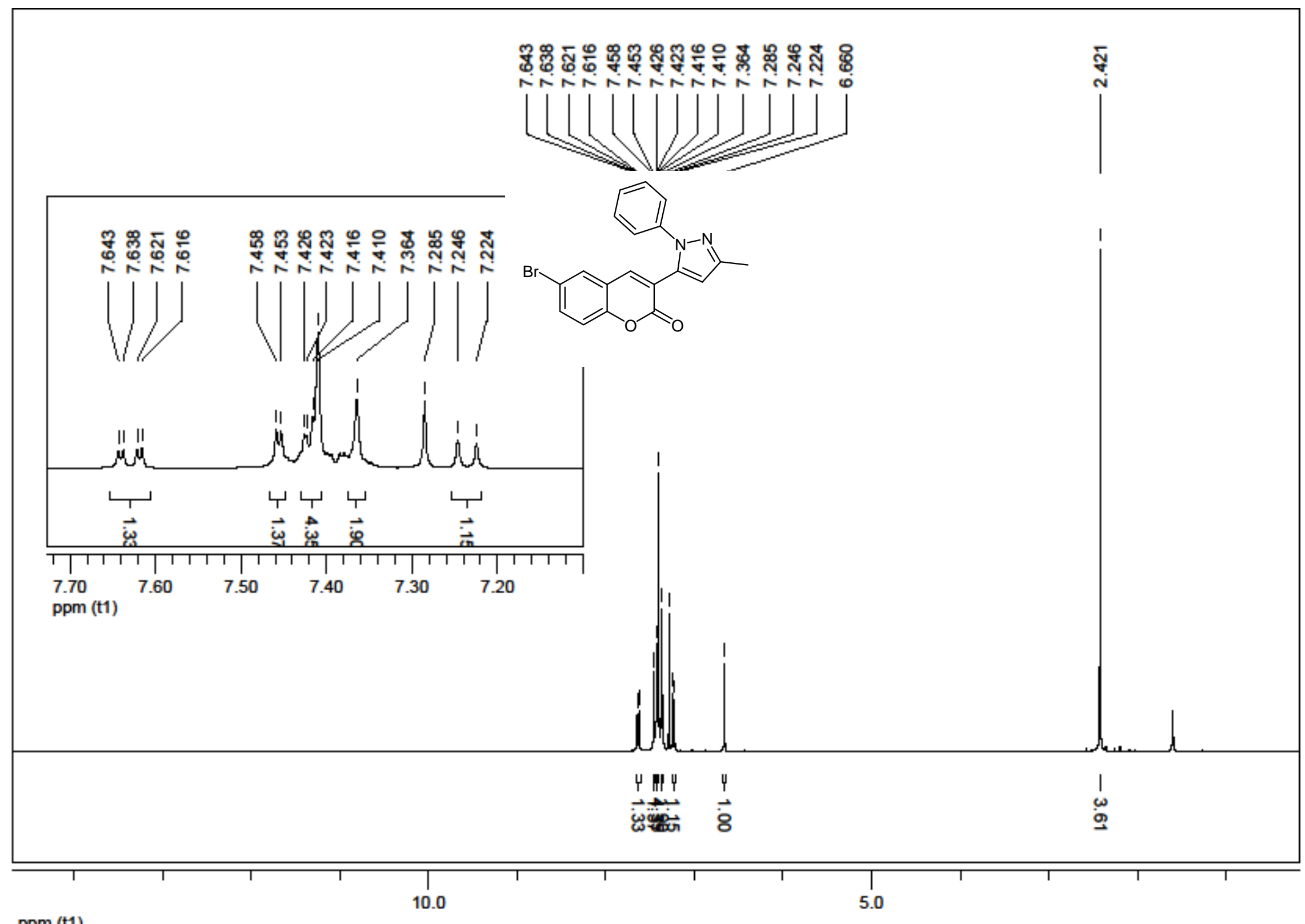

ppm (t1)

Figure $\mathrm{S} 3{ }^{1} \mathrm{H}$ NMR of $\mathbf{3 b}$ 


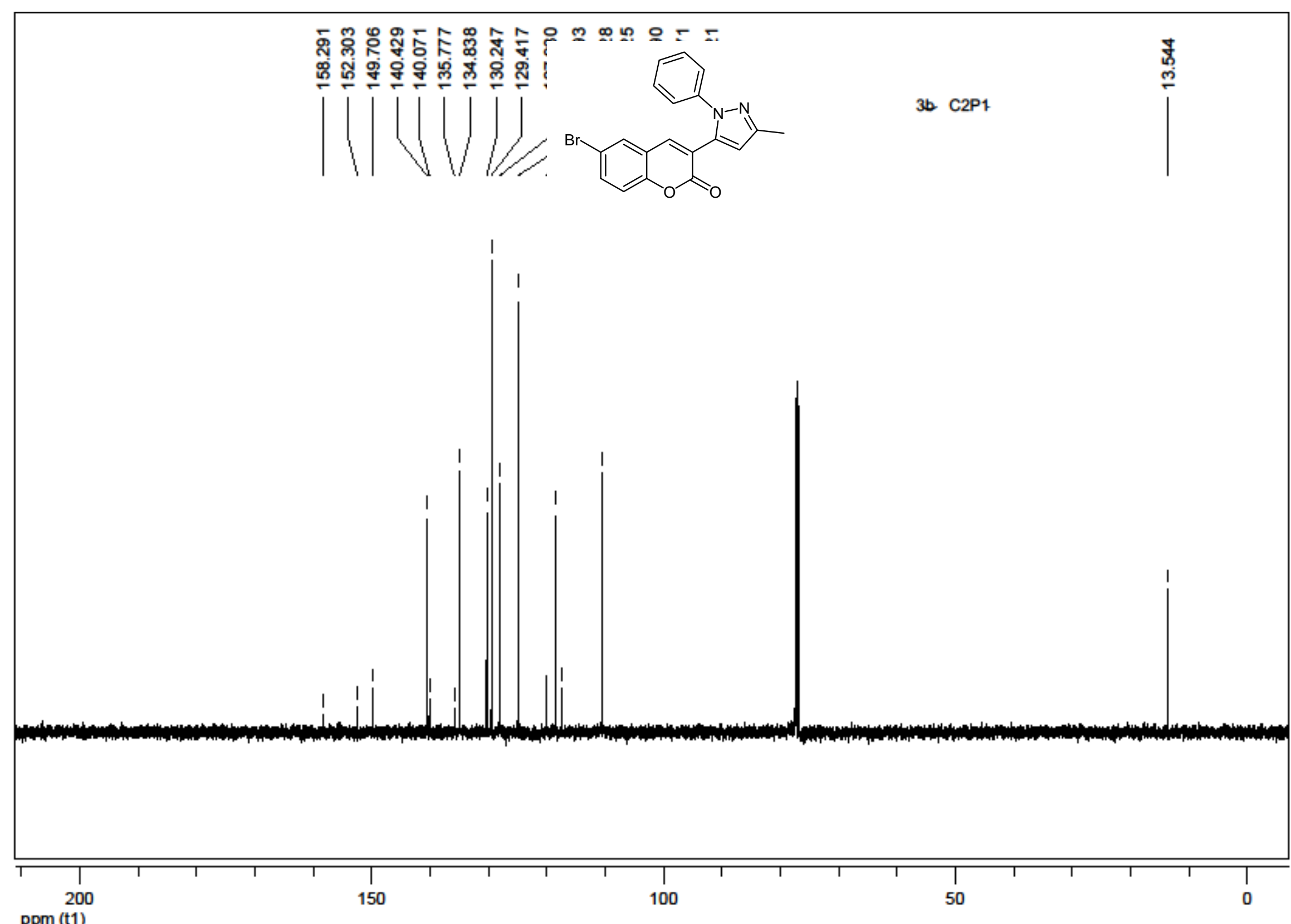

Figure S4 ${ }^{13} \mathrm{C}$ NMR of $\mathbf{3 b}$ 


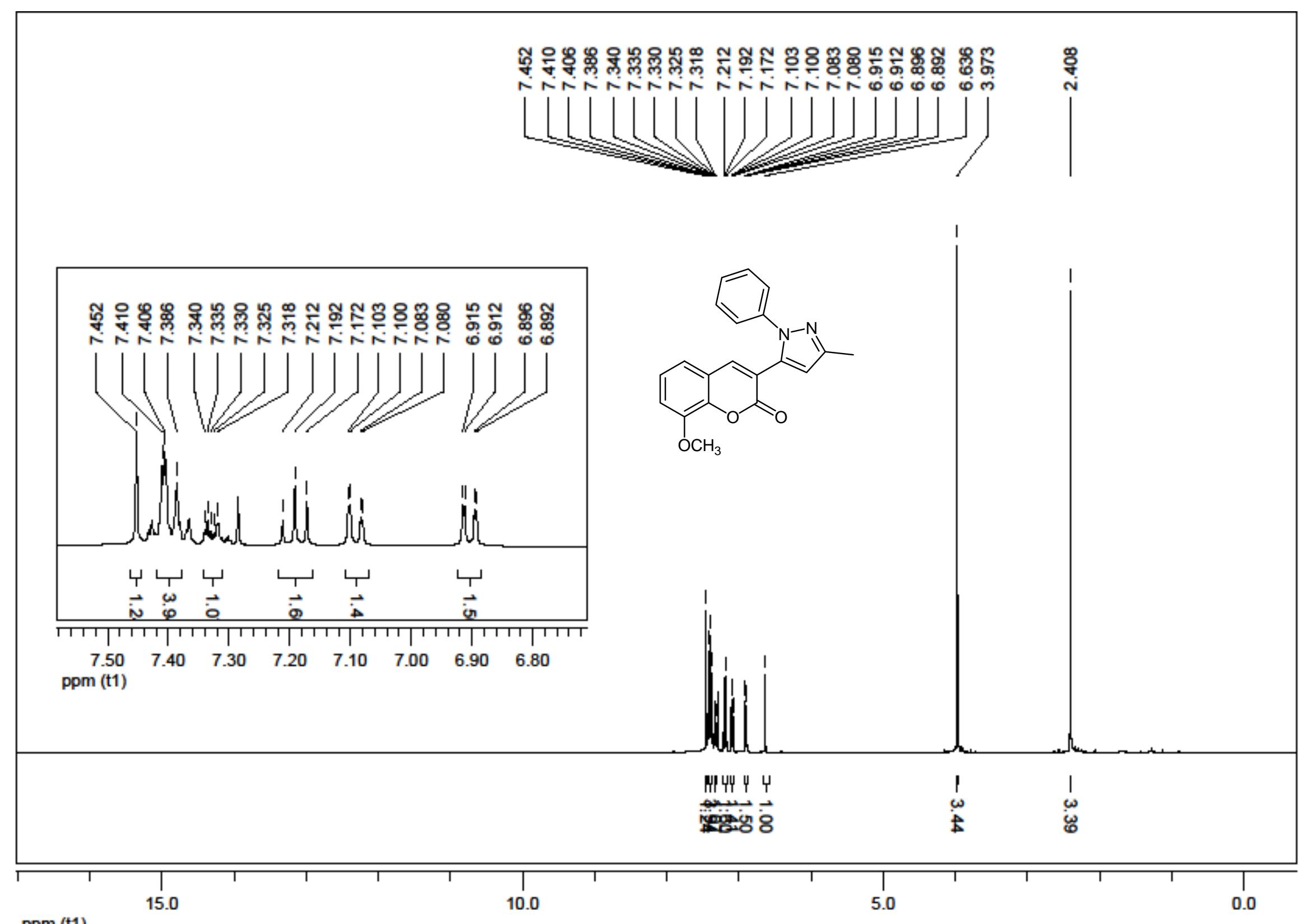

ppm (t1)

Figure S5 ${ }^{1} \mathrm{H}$ NMR of $3 \mathrm{c}$ 


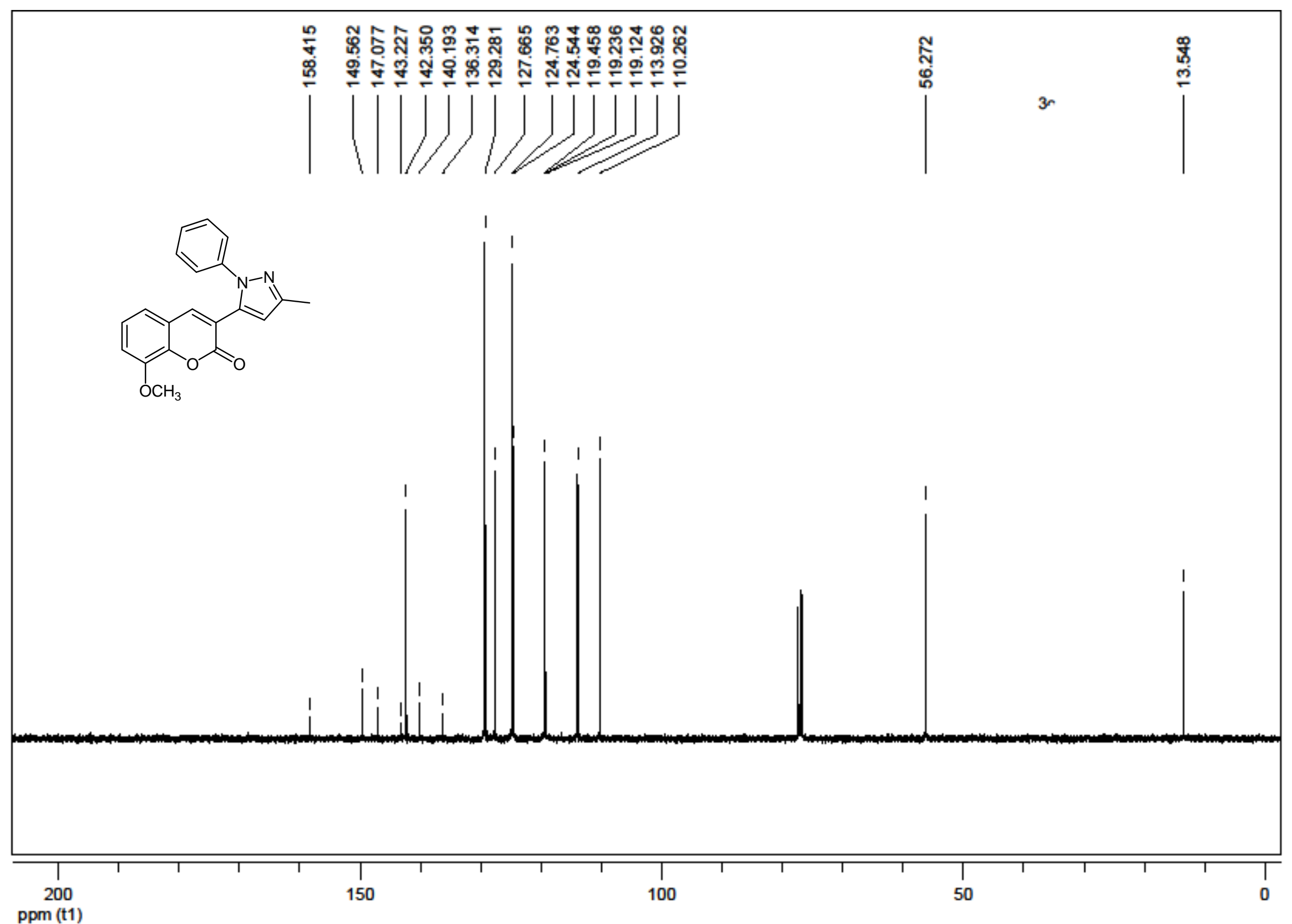

Figure $\mathrm{S} 6{ }^{13} \mathrm{C}$ NMR of 3c 


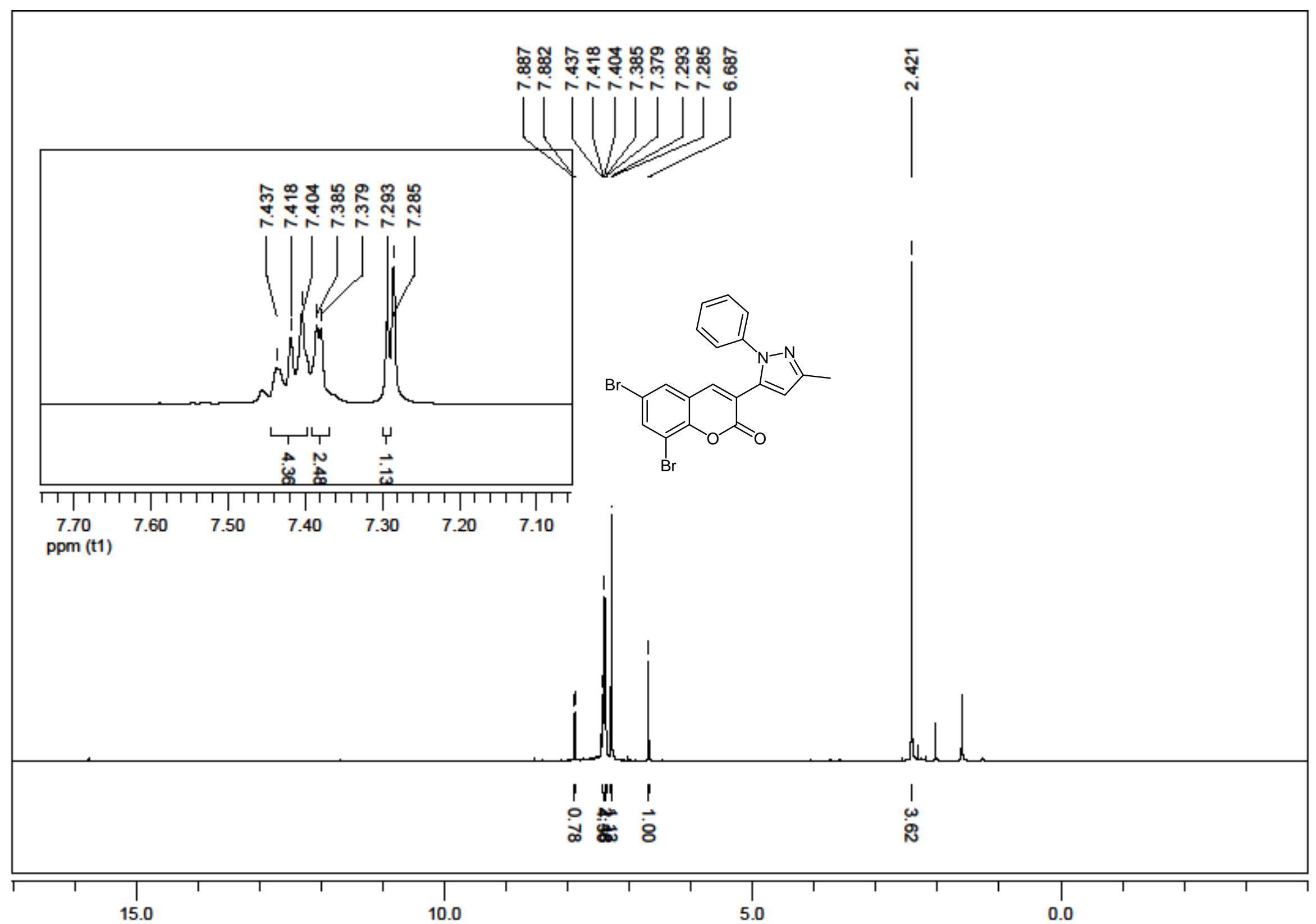

ppm (t1)

Figure S7 ${ }^{1} \mathrm{H}$ NMR of 3d 


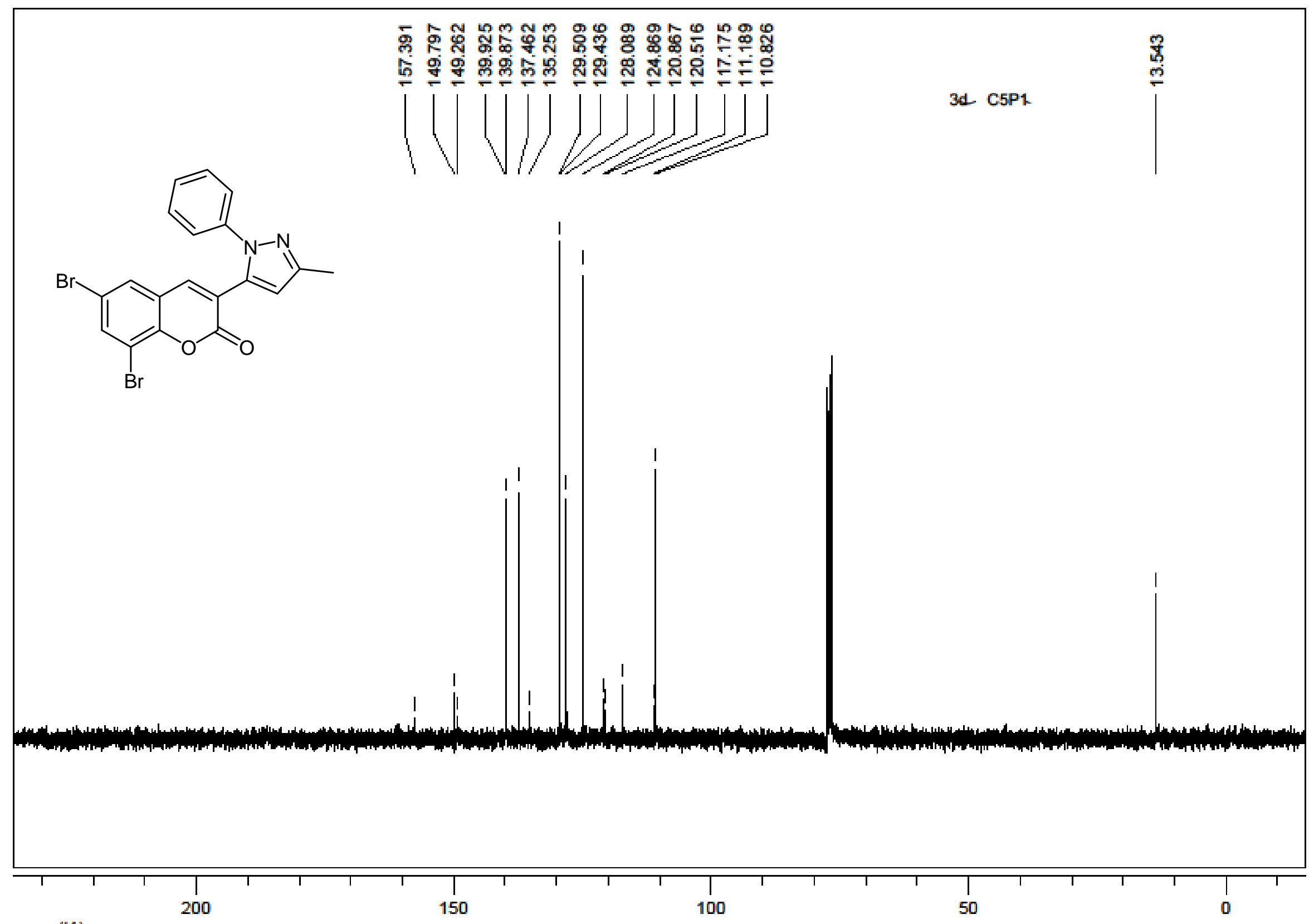

ppm (t1)

Figure S8 ${ }^{13} \mathrm{C}$ NMR of 3d 


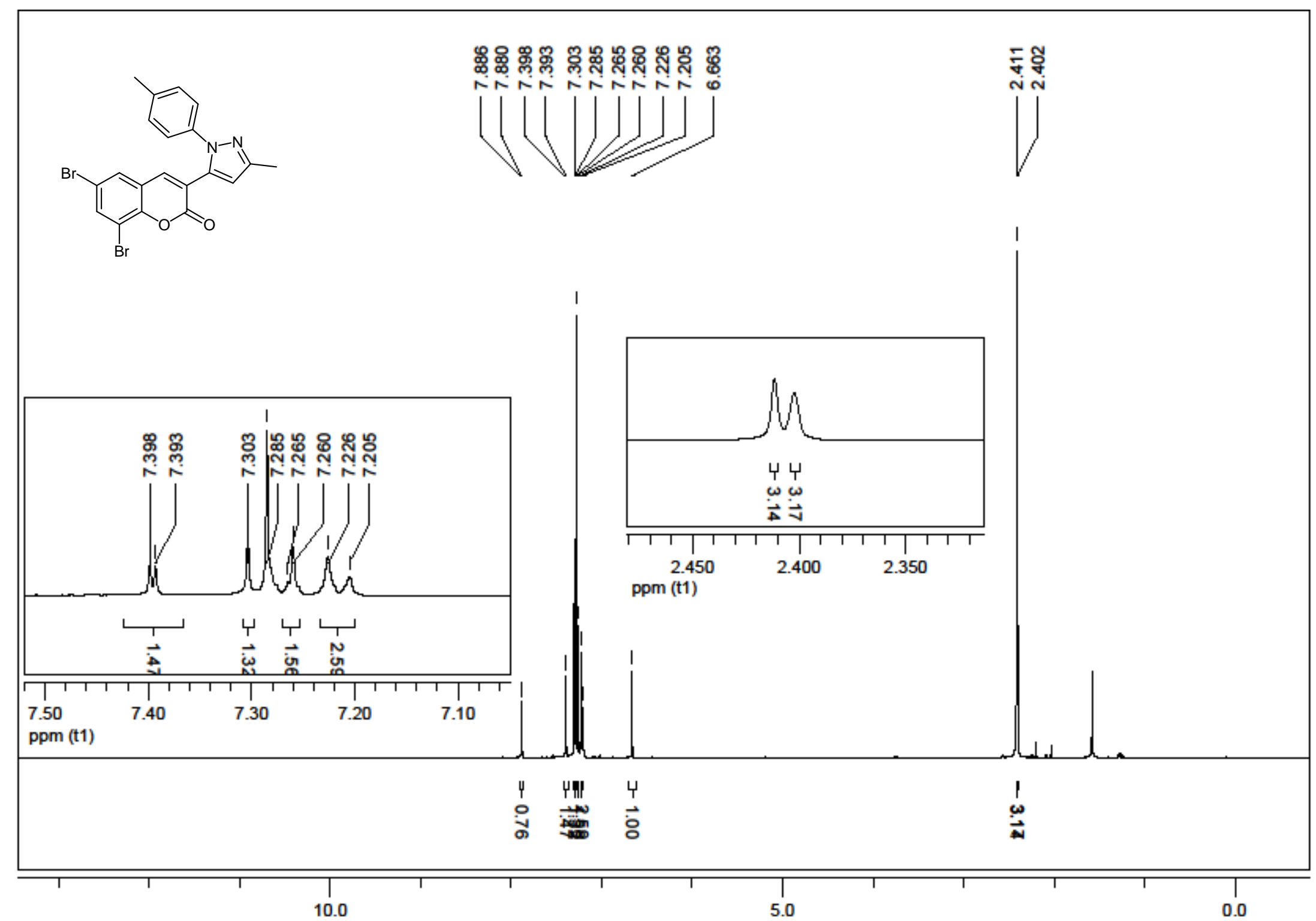

ppm (t1)

Figure S9 ${ }^{1} \mathrm{H}$ NMR of $3 \mathbf{e}$ 


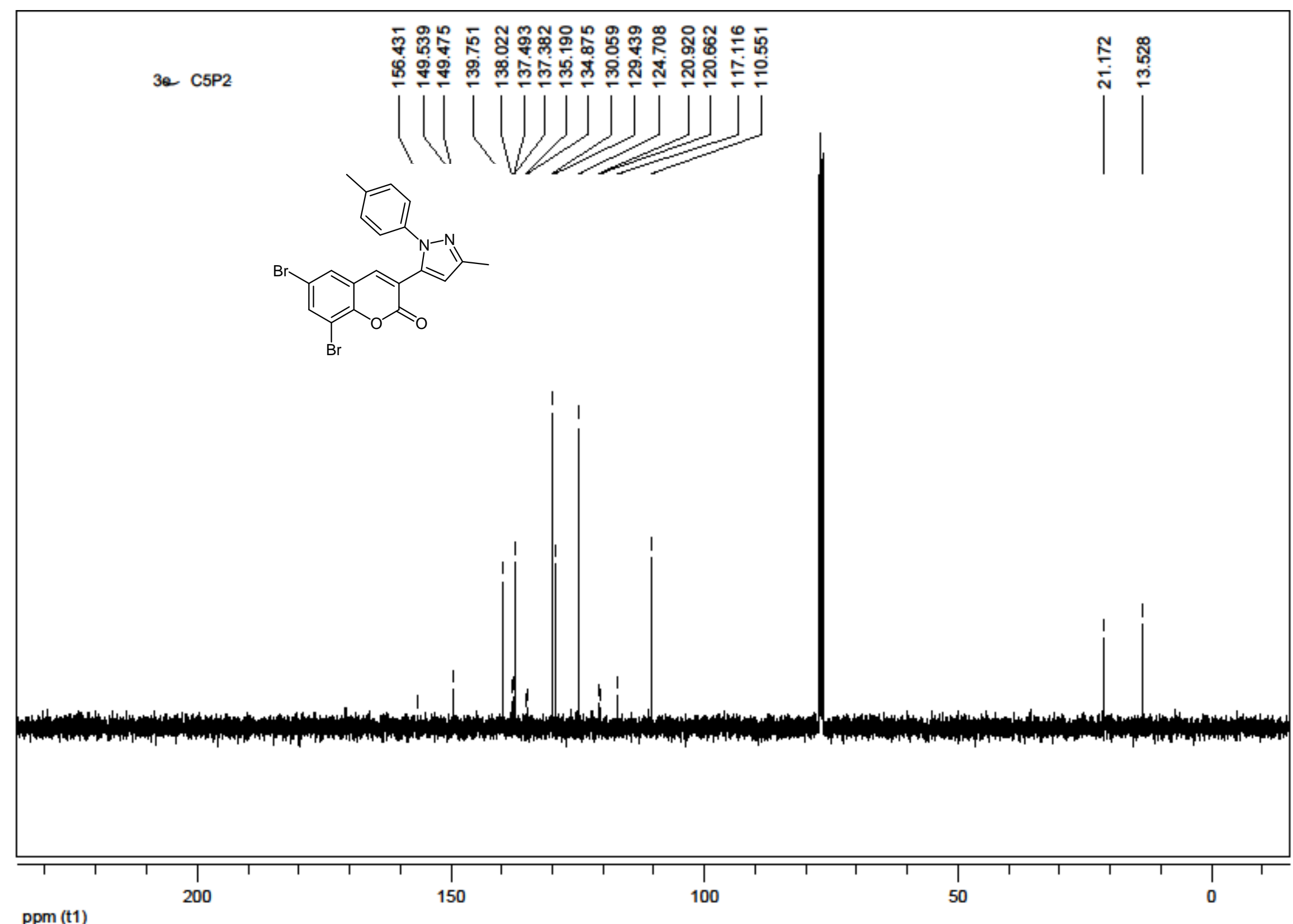

ppm (t1)

Figure S10 ${ }^{13} \mathrm{C}$ NMR of $3 \mathbf{e}$ 


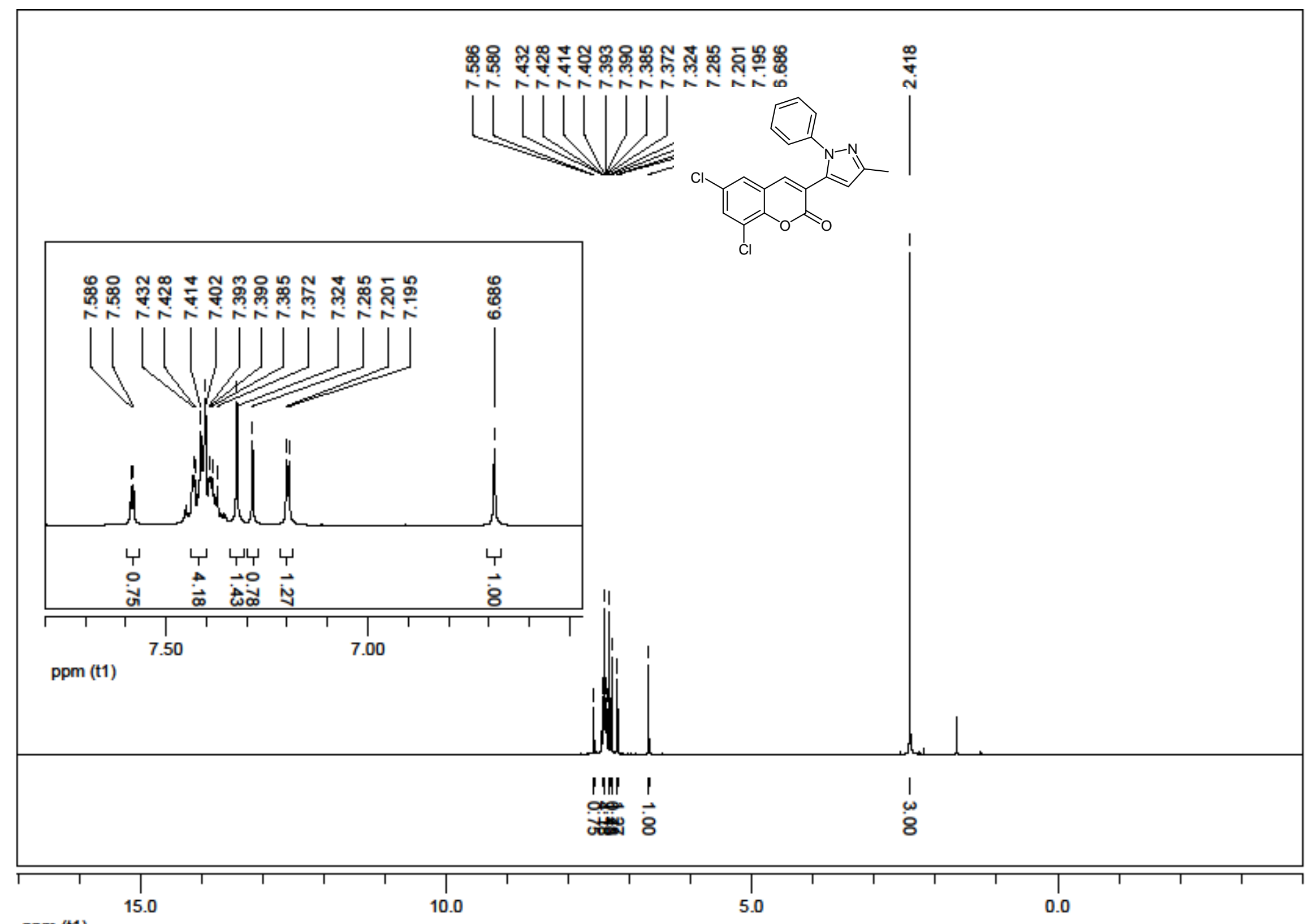

ppm (t1)

Figure S1 $1{ }^{1} \mathrm{H}$ NMR of $\mathbf{3 f}$ 


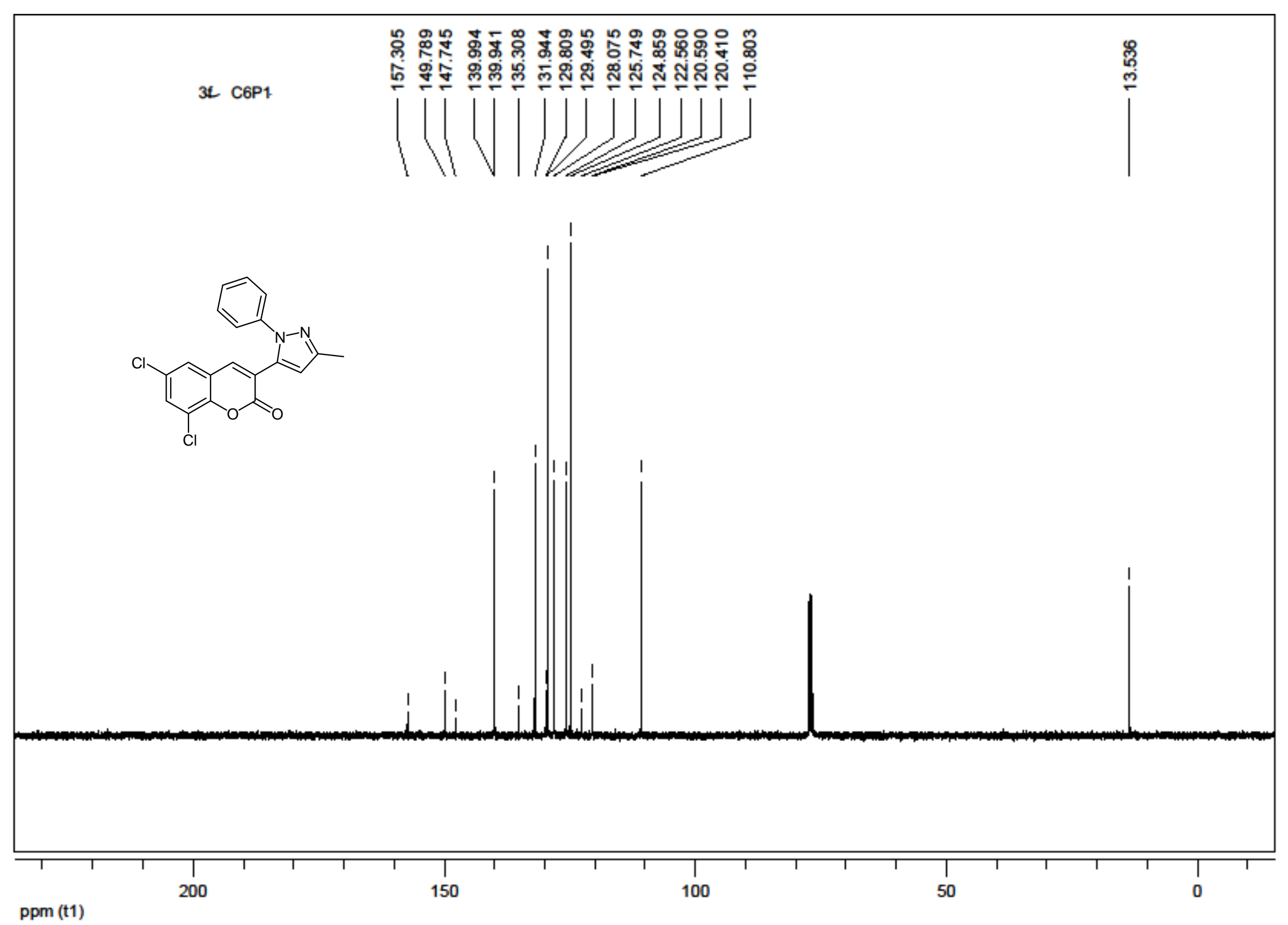

Figure S12 ${ }^{13} \mathrm{C}$ NMR of $3 \mathbf{f}$ 


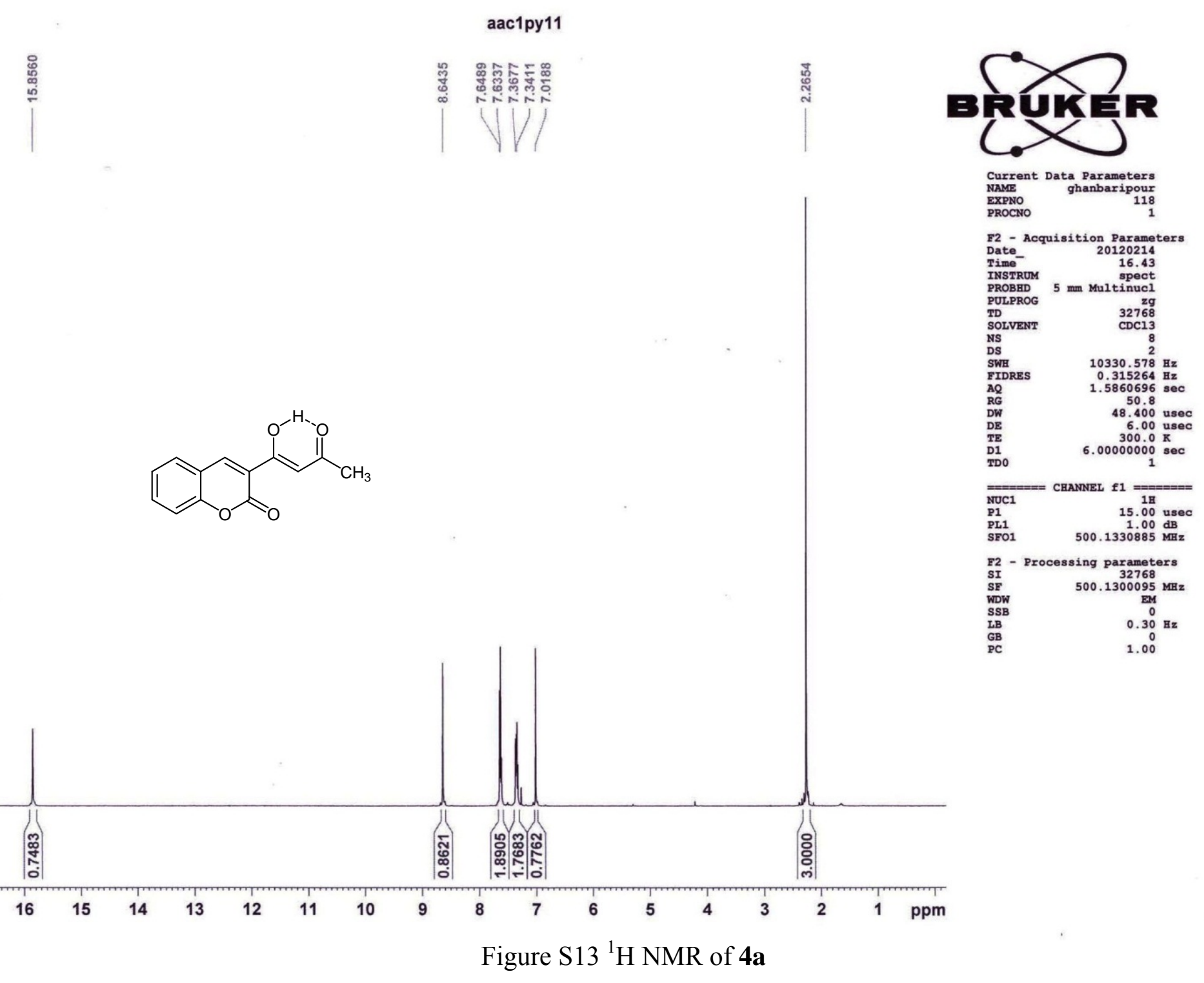




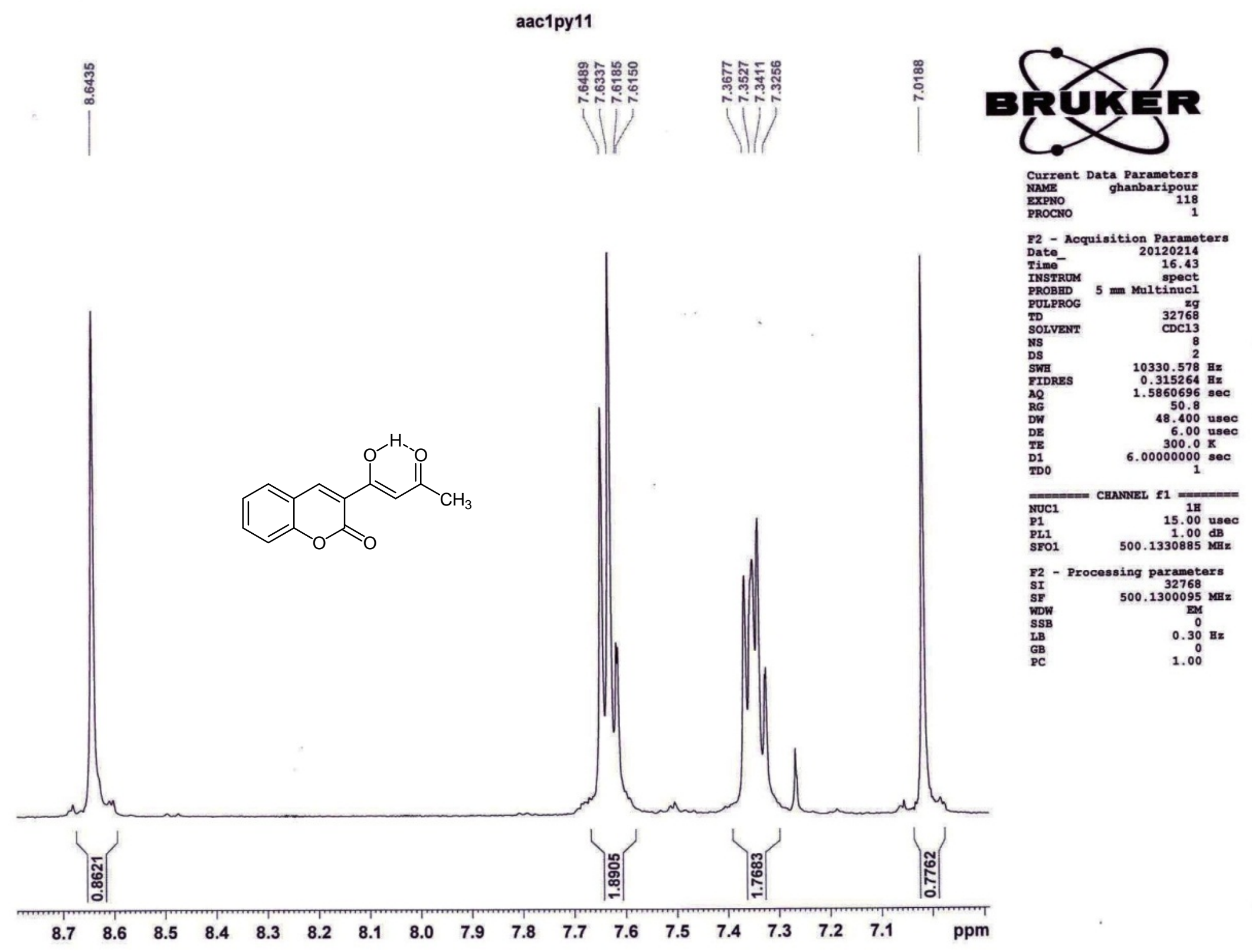

Figure S14 ${ }^{1} \mathrm{H}$ NMR of $\mathbf{4 a}$ (expand) 


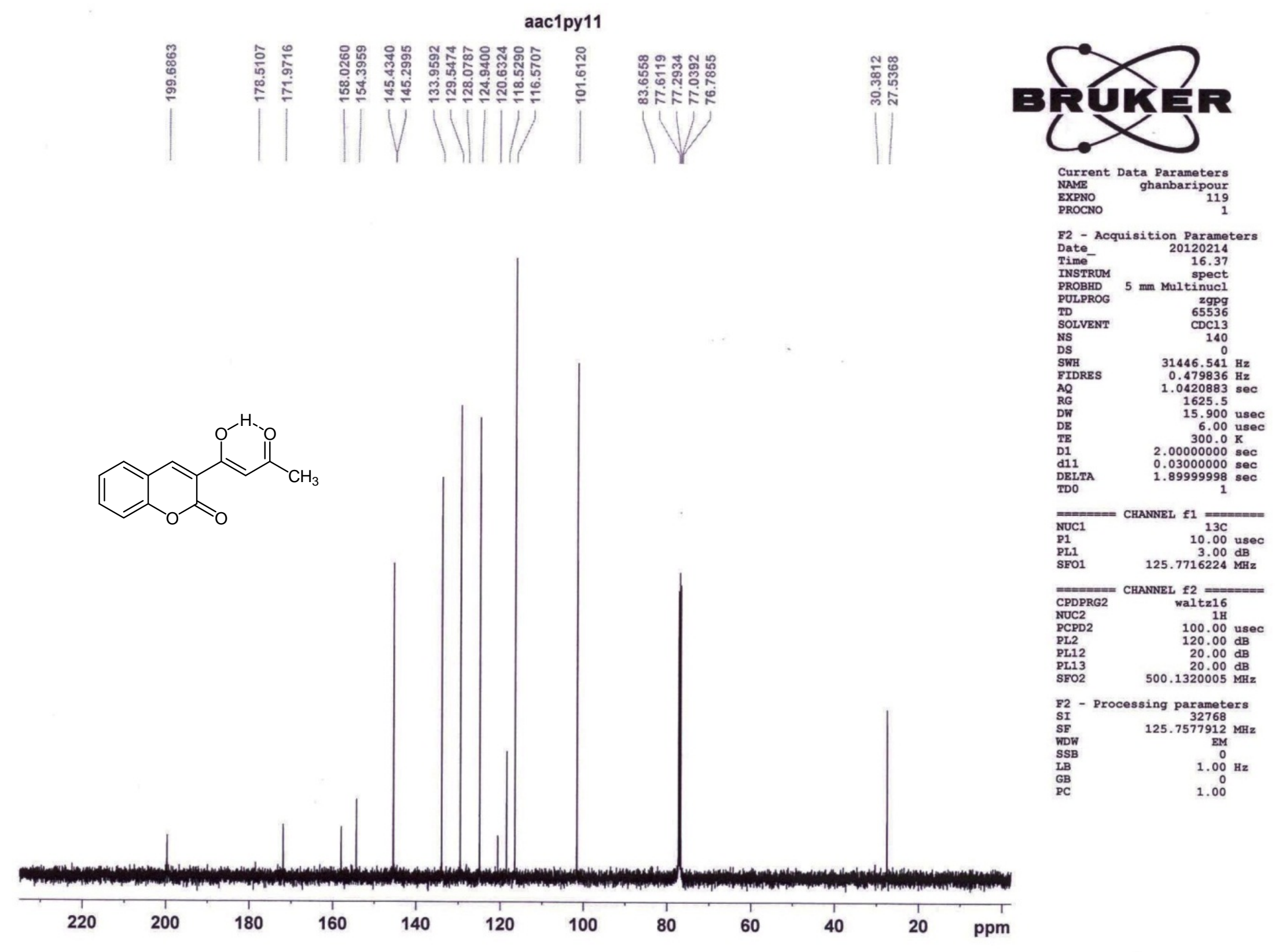

Figure S15 ${ }^{13} \mathrm{C}$ NMR of $4 a$ 